\title{
IE RECUL DU FRANÇAIS COMME LANGUE DES SCIENCES
}

\author{
JACQUELINE BOSSÉ-ANDRIEU \\ UNIVERSITÉ D'OTTAWA
}

\section{CRIS D'ALARME}

Depuis plusieurs années, l'utilisation croissante de l'anglais dans la communication technique et scientifique ne cesse de susciter des inquiétudes et des cris d'alarme. Rapports, colloques, articles, etc., se multiplient pour attirer l'attention sur la menace qui pèse sur le français et pour remettre en question la place que peut encore occuper cette langue dans la communication scientifique à l'échelle internationale. Déjà, en juin 1980, s'était tenu à Paris, à l'Université d'orsay, un colloque intitulé "L'anglais : langue scientifique française". Dix ans après, en janvier 1990, à Paris, a été organisé un forum intitulé "Quelles langues pour la science?" Une décennie s'est écoulée entre les deux événements, et le constat est toujours le même : la prépondérance de l'anglais met en péril le français. Comme l'a dit le ministre français de la Francophonie au dernier forum, "la situation du français est extrêmement préoccupante" puisque, dans presque toutes les disciplines scientifiques - il y aurait quelques exceptions comme les mathématiques -, la plupart des scientifiques de langue française publient le résultat de leurs travaux en anglais dans des revues anglo-saxonnes ou françaises. 
L'exemple le plus frappant - et qui a peut-être servi de détonateur - est celui que nous a récemment donné l'Institut Pasteur. On se souvient que, le 4 avril 1989, les dirigeants de cet institut ont annoncé leur intention de publier les Annales sous la forme de revues portant des titres anglais (Research in ...). quelles que soient les raisons invoquées (un titre français laisse penser que le contenu d'une revue est majoritairement dans cette langue; les revues de l'Institut doivent apparaitre comme des revues internationales ouvertes aux chercheurs de tous les pays), cette décision a provoqué un tollé; qualifiée d'"absurde" et de "scandaleuse" en France comme dans d'autres pays francophones (en particulier au Canada), elle a été vue comme le symbole de la démission des scientifiques devant l'hégémonie croissante de l'angloaméricain.

Finalement, la nouvelle du projet d'anglicisation d'une revue française prestigieuse et centenaire a fait l'effet d'une bombe et a contribué à réveiller les consciences. Si, pour certains, le recul du français est un phénomène irréversible auquel il faut se résigner, pour d'autres il est encore temps de prendre des mesures pour enrayer le déclin du français dans les sciences. Après avoir examiné les faits puis leurs causes et conséquences, nous verrons quels remèdes sont proposés pour préserver le français comme langue des sciences.

\section{LES FAITS}

Alors que, dans les sciences sociales et humaines, la France jouit d'une réputation internationale (même si les lecteurs étrangers ont recours à des traductions) - le fait qu'en 1988 le prix Nobel de sciences 
économiques ait été décerné à Maurice Allais pour des travaux écrits et publiés en français le prouve -, la situation est bien différente dans d'autres disciplines où les chercheurs s'expriment presque exclusivement en anglais. Selon l'Université des réseaux d'expression française (UREF), la plupart des mathématiciens francophones, comme les médecins, les juristes et les agronomes, s'expriment et publient toujours le plus souvent en français, mais les physiciens, les chimistes et les biologistes utilisent de plus en plus l'anglais. De l'aveu de Pierre Joliot, professeur au Collège de France, en biologie il n'est pas question de publier dans une langue autre que l'anglais (Le Devoir, 12 oct. 90). Et cette tendance serait irréversible. Plusieurs revues au titre français (comme la Nouvelle Revue française d'hématologie) ne publient que des articles en anglais. De nouvelles revues (comme Fundamental \& Clinical Pharmacologyl adoptent au départ un titre anglais et ne publient que des articles rédigés en anglais. D'autres abandonnent leur titre français pour prendre un titre anglais (ex. : European Journal of Mechanics au lieu de Journal de mécanique théorique et appliquée). Bref, en France, moins de $50 \%$ des articles sont rédigés en français.

Pour le Québec, la situation n'est guère plus reluisante. En 1970, 708 des articles des publications québécoises étaient rédigés en anglais; ce chiffre s'est élevé à 73\% entre 1980 et 1983. Et, en ce qui concerne les sciences physiques, les mathématiques et la biologie, $85 \%$ des publications sont rédigées en anglais.

Les tableaux qui suivent sont éloquents. 
I Situation dans le monde en $1986^{1}$

a) Langues

Sur l'ensemble des publications scientifiques

- $65 \%$ sont rédigées en anglo-américain

$-12 \%$ sont rédigées en russe

$-9,8 \%$ sont rédigées en français

$-7,6 \%$ sont rédigées en allemand

b) Pays

Production scientifique (en quantité de publications)

- 47\% de la production vient des pays d'expression anglo-saxonne

- 7\% de la production vient des pays d'expression française

- 78 de la production vient des pays d'expression allemande

Source : Haut Conseil de la francophonie (1987, 159)

II Production mondiale (par pays en 1982)

Etats-Unis : $37 \%$ des publications (= environ $3 \%$ par rapport à 1973)

Royaume-Uni : 8,3응 des publications (= environ 10 z par rapport à 1973)

Japon : 7,3\% des publications (= environ + $40 \%$ par rapport à 1973 )

RFA : 6,2\% des publications (= environ + 3응 par rapport à 1973)

France : 5,06\% des publications (= environ $10 \%$ par rapport à 1973 )

Canada : $4 \frac{\%}{8}$ des publications (= environ - $8 \%$ par rapport à 1973)

Source : CHI - NSF ${ }^{2} /$ Haut Conseil de la francophonie (1987, 189-190) 
II Situation au Québec (entre 1980 et 1983)

Pourcentage des publications en langue anglaise des chercheurs rattachés aux 4 universités francophones et aux 6 instituts de recherche paragouvernementaux : 73\%

a) Université de Montréal

: $68 \%$

Université du Québec

$: 42 \frac{8}{8}$

Université de Sherbrooke

Source : Sénéchal/CLF ${ }^{3}(1989,6)$

: $90 \%$

b) Instituts de recherche : $80 \%$

Institut de recherche en énergie d'Hydro Québec et Institut du cancer : $92 \%$

Source : CLF $(1986,33)$

c) Domaines

Sciences physiques et mathématiques : $90 \%$

Sciences biologiques et médicales : 75\%

Sciences humaines et sociales: $27 \%$

Source : CLF $(1986,31)$

IV Situation en France

Proportion d'articles rédigés en francais :

Sciences médicales et

$$
\text { en } 1980 \text { en } 1974
$$

biologiques

$57,9 \frac{8}{8}$

$69,5 \%$

Physique et sciences de

l'ingénieur

Chimie

$21,2 \frac{8}{8}$

$40,4 \%$

Mathématiques

43,38

80,4 웅

$74,6 \%$

Source: ISI/CLF (1983, III, 179)

$93,5 \%$ 
Pays de la francophonie (1980)

Proportion d'articles publiés

$\begin{array}{lll} & \text { en français } & \text { en anglais } \\ \text { France : } & 2 / 3 & 1 / 3 \\ \text { Canada et Québec : moins de } 1 / 5 & \text { plus de } 4 / 5 \\ \text { Belgique : } & 1 / 3 & 2 / 3 \\ \text { Suisse : } & \text { moins de } 1 / 5 & 1 / 2 \\ \text { Source : CLF (1986, 36) } & \end{array}$

Alors que, naguère, un quart de I'information scientifique et technique (I.S.T.) était produite en français (CLF, 1986, 5), la place du français ne cesse actuellement de diminuer, surtout dans les domaines où les retombées socio-économiques des travaux peuvent être importantes - ce qui explique son maintien dans les sciences humaines et sociales. C'est l'anglais qui est le plus souvent utilisé, même dans les revues plurilingues d'associations européennes spécialisées, et même dans les revues publiées en France et dans les pays francophones. Les scientifiques francophones choisissent fréquemment de publier en anglais des articles qui paraitront dans des revues françaises et, si ce sont des articles de haut niveau, ils cherchent à les publier dans une revue américaine.

En ce qui concerne la langue de travail utilisée dans les congrès et colloques scientifiques, là encore c'est le plus souvent l'anglais - même en France, même si $80 \%$ des participants sont francophones et même si la langue officielle est le français. (Un conférencier parlant français voit la salle se vider!)

Un rapport présenté en 1980 par la section française de l'Association internationale des parlementaires de langue française (AIPLF) fourmille d'exemples 
attristants et choquants : colloques annoncés en anglais, colloques tenus en France où seul l'anglais était accepté, enseignement de certaines matières, dans des instituts nationaux, en anglais (par des professeurs français s'adressant à des étudiants français), chercheurs incités, par des organismes nationaux, à publier en anglais. Dans les congrès européens regroupant des participants des pays européens, l'anglais est la langue recommandée. Au Québec, entre 1980 et 1983, les chercheurs rattachés aux universités francophones et aux instituts de recherche ont présenté $54 \%$ de leurs communications en anglais; la proportion atteint les deux tiers pour les mathématiciens et les physiciens (CLF, 1986).

Il est donc indéniable que l'anglais est, à l'heure actuelle, la langue de communication de la plupart des scientifiques, y compris des scientifiques francophones. cet état de choses s'explique de plusieurs façons.

\section{SUPREMATIR DES ETATS-UNIS}

La première raison de la prépondérance de l'anglais dans la langue de I'I.S.T. est la suprématie des États-Unis dans les domaines politique, économique et scientifique. En ce qui concerne la recherche et le développement, c'est aux États-Unis que s'effectue la moitié de l'activité scientifique mondiale, grâce à des budgets distribués généreusement, entre autres par de nombreuses institutions privées.

C'est aussi aux Etats-Unis que se concentrent une grande partie de I'I.S.T. et les revues scientifiques de haut niveau. Il est donc naturel que des chercheurs francophones viennent se former - et même parfois s'établir - dans le pays qui produit le plus grand nombre de Prix Nobel. 


\section{"PUBLISH OR PERISH"}

Tout chercheur désirant être lu, reconnu et donc cité, il lui importe que le résultat de ses travaux soit publié dans des revues prestigieuses. Or, la base de données la plus considérable - et celle qu'utilisent tous les chercheurs - est celle de l'Institute for Scientific Information (ISI) dirigé par Eugene Garfield, qui cote, selon des criteres objectifs, revues, articles et auteurs. L'ISI, institut privé de Philadelphie qui a des impératifs économiques, techniques et scientifiques, dépouille près de $90 \%$ de la littérature scientifique et technique produite dans le monde (plus de 3200 revues) et public les Current Contents (hebdomadairement) pour chacun des grands domaines de recherche, le Science Citation Index et le Social Citation Index, qui classent les auteurs et les articles selon le nombre de citations dont ils ont fait l'objet. Même si l'impartialité de l'ISI est parfois contestée par des chercheurs non anglophones qui accusent cet institut de privilégier les articles et les revues de langue anglaise, il $n^{\prime e n}$ reste pas moins que les milieux scientifiques de tous les pays attachent une grande importance à ses données. Les chercheurs savent que, si leurs articles sont stockés dans la banque de I'ISI, ils jouissent d'une audience potentielle considérable. Les francophones, pour être plus sûrs d'être cités, publient donc en anglais. On comprend encore mieux cette tendance quand on sait que le prix Nobel et d'autres grands prix internationaux sont adjugés sur la base du nombre de citations dans les revues américaines ( $L$ 'Arbre à palabre des francophones, 135).

D'un autre côté, certains chercheurs francophones choisissent les revues américaines, car ils reprochent à de nombreux périodiques de langue française leur 
piètre qualité, leur faible diffusion, leur multiplicité dans un même secteur de recherche et leur présentation médiocre (CLF, 1986). Enfin, ils estiment que leur rôle essentiel est de faire progresser la science - et donc de faire connaitre leurs travaux - et qu'il faut dissocier rayonnement culturel et rayonnement scientifique. A leur avis, publier exclusivement en français, c'est limiter considérablement l'influence de leurs travaux sur la communauté scientifique, puisque les revues américaines citent rarement les articles rédigés en français. Sans compter que le résultat de leurs recherches risque d'être utilisé et subtilisé - par d'autres qui les traduisent sans en citer l'auteur, comme un ingénieur en linguistique établi au Canada en a fait l'amère expérience (Lapierre et Marchand, 1989).

\section{PARTI PRIS, AMOUR-PROPRE ET SNOBISME}

En outre, les organismes nationaux ont contribué au recul du français en décourageant indirectement les chercheurs de publier dans cette langue. Le prestige de l'anglais est en effet tel que les évaluateurs appartenant à des organismes et à des instituts de recherche français sont accoutumés à prendre en considération essentiellement les travaux publiés dans des revues prestigieuses - donc américaines - et à évaluer plus généreusement les travaux publiés en anglais (Laplane, 1986); selon I'AIPLF (1980), les auteurs qui utilisent l'anglais sont favorisés lors du recrutement et de la promotion de chercheurs. On les tient pour des chercheurs d'audience internationale. Qui plus est, les francophones qui s'expriment en anglais ont le sentiment d'appartenir à une élite. Comme l'a dit un universitaire français : "Un Anglais est fier d'entendre quelqu'un capable de parler sa 
langue. Un Français est fier de parler une autre langue que la sienne!" (CLF, 1983, III, 241). Résultat de ce snobisme : alors que les auteurs anglais et américains ne se donnent que rarement la peine de lire les travaux rédigés dans une autre langue que la leur, les chercheurs français - à l'instar des Français qui émaillent leur conversation de mots anglais - n'hésitent pas à rédiger ou à parler en anglais.

\section{QUESTION DE LANGUES}

Par ailleurs, on peut penser que les subtilités grammaticales et orthographiques du français, langue sclérosée depuis la Révolution, sont de nature à en freiner la diffusion. Le français est en effet une langue difficile, souvent à la traine de l'anglais en ce qui concerne les néologismes, et qui, surtout, "souffre" d'une longue tradition de purisme et "d'amour du beau langage". Et l'auteur qui n'observe pas les règles de la grammaire ou les décisions de l'Académie française s'expose a souverain mépris de ses compatriotes. D'où la tentation de s'exprimer en anglais - même si on s'exprime imparfaitement dans cette langue. Quant aux chercheurs étrangers, ils sont rebutés par le niveau de connaissances linguistiques exigé en français (Hagège, 1987). Par contre, le monde scientifique anglo-saxon admet un usage pragmatique de la langue et, si le message passe, excuse les imperfections de nature linguistique. En outre, l'utilisation de l'anglais est encouragée par le fait qu'en anglais la grammaire, comme l'affirme un professeur belge (CLF, 1983, III, 311), est relativement simple et la syntaxe plus souple que celle du français. 


\section{NECESSITE D'UNE LANGUE INTERNATIONALE}

Une dernière raison, mais non la moindre, est la nécessité que ressent le monde scientifique de parler une langue commune. Jusqu'au milieu du XVII siècle, les érudits des pays d'Europe qui se rencontrent parlent latin. On sait que Newton publie les principes mathématiques de philosophie naturelle (1687) en latin, et en anglais son Traité d'optique (1704). Ensuite, pendant deux siècles, le français, l'anglais et l'allemand se font concurrence. Puis l'anglais, depuis le début du $\mathrm{xx}^{\mathbf{e}}$ siècle, occupe la première place dans un domaine où les résultats des recherches, publiés dans des documents dont le nombre grandit sans cesse, doivent circuler rapidement et mondialement (Hagège, 1987). Et, pour beaucoup de chercheurs, c'est l'avancement de la science, la possibilité d'échanger des résultats qui priment et non la langue utilisée, qui est un simple outil.

Diverses raisons concourent donc à la promotion de l'anglais comme langue internationale de I'I.S.T. Cependant, cette situation, aux multiples conséquences, engendre des inquiétudes.

\section{UN CERCLE VICIEUX}

La prépondérance de l'anglais dans I'I.S.T. et la suprématie des états-Unis dans le monde sont si grandes qu'elles exercent une fascination croissante, qui entraine à son tour une utilisation croissante de l'anglais. Cette langue devient non seulement la langue de I'I.S.T., mais aussi la langue de communication internationale. L'anglais est parlé dans le monde entier (banques, hôtels, aéroports, etc.). C'est déjà la langue semi-officielle du Marché commun (et on 
songerait à faire de Bruxelles un district dont l'anglais, le français et le néerlandais seraient les langues officielles). L'engouement pour l'anglais s'étend aux communications personnelles. Lapierre et Marchand (1989) citent le cas d'une lettre envoyée en anglais par l'Institut Pasteur à un chercheur canadien francophone. P. de Saint Robert (1986) donne d'autres exemples d'aberrations de ce type imputables à des organismes publics ou à des services diplomatiques français.

Cette acceptation de l'anglais comme langue de communication et langue de I'I.S.T. engendre un autre cercle vicieux. Plus le nombre de travaux rédigés en anglais croit, plus les chercheurs francophones délaissent le français - et plus ils privent certains de leurs compatriotes du fruit de leurs recherches. $\mathrm{Ce}$ qui renforce encore la supériorité des États-Unis dans la recherche scientifique et l'hégémonie de l'anglo-américain à l'échelle internationale.

\section{APPAUVRISSEMENT DU FRANÇAIS ET DE LA PENSÉE FRANÇAISE}

Le recul du français dans I'I.S.T. internationale conduit, dans les pays francophones, à une réduction de l'usage de cette langue dans l'enseignement et dans les milieux de travail; on a déjà signalé l'anglicisation des troisièmes cycles en France où les livres d'enseignement universitaire en français manquent. Par ailleurs, ce recul sur les deux fronts - international et national - provoque un appauvrissement de la terminologie et de la langue françaises. Comme le note J. Lacant (1989) en rapportant les réflexions du professeur Drapeau de Montréal, qui s'est insurgé contre la décision de l'Institut pasteur, "exclure le 
français de nos grandes revues scientifiques, c'est dissuader de former des mots français pour les notions nouvelles, c'est en faire une langue archaique, impropre à la modernité". Faire de l'anglais la langue internationale de I'I.S.T., c'est aussi favoriser l'anglicisation du français - les sciences devenant ainsi "le cheval de Troie de l'anglicisation" (J.-P. Rogel cité par Lapierre et Marchand (1989)) -, anglicisation qui s'accélérera lorsque le support imprimé sera remplacé par le support informatique, puisque presque toutes les banques de données sont en anglais. L'anglais influence déjà l'orthographe, le lexique, la syntaxe et le style de la langue technique informatique (Koechlin, 1988).

Certains redoutent, en outre, que les modes de pensée en français s'appauvrissent (CLF, 1986), les francophones étant obligés de modeler leur pensée sur celle des anglophones et d'"intérioriser des modes de raisonnement appartenant à une autre culture" (Cassen, 1985). Cette réflexion, qui vise plus particulièrement l'informatique, s'applique également aux domaines techniques et scientifiques. Et certains voient là un risque de nivellement généralisé, d'uniformatisation des valeurs (Decaux, 1990).

Enfin, les chercheurs francophones qui s'expriment dans une langue qui n'est pas la leur sont désavantagés par rapport aux anglophones. Ainsi, sur 100 chercheurs québécois interrogés, $70 \%$ estiment qu'ils sont moins efficaces lorsqu'ils s'expriment dans une langue seconde (CLF, 1983, 13).

\section{"BASIC ENGLISH" OU "PIDGIN YANKEE"}

En ce qui concerne l'anglais utilisé par les chercheurs non anglophones, tout le monde convient qu'il est loin 
d'être idiomatique. Selon certains, la langue universelle des scientifiques ressemblerait plus à un "pidgin yankee" (Lévy-Leblond, 1990), à un anglais simplifié, qu'à la langue anglaise ou américaine; conséquence : l'anglais, langue internationale "beaucoup plus bredouillée que parlée dans les organismes internationaux", perd ainsi "son âme" (CLF, 1983, III, 240). Mais, il faut l'avouer, pour beaucoup, ce n'est pas là une considération importante, l'essentiel étant de communiquer.

\section{SITUATION IRREVERSIBLE?}

Cette situation provoque, de la part des chercheurs francophones, des réactions différentes. Pour les uns, il faut s'y résigner : la science a besoin d'une langue universelle qui est, à l'heure actuelle, l'anglais. D'ailleurs, affirment certains, on ne peut parler de recul du français, $n i$ de situation catastrophique du français. Cette langue, disent-ils, occupe depuis longtemps dans les sciences la deuxième place et se maintient dans cette position. De plus, la plupart des scientifiques du monde entier liraient le français dans leur discipline (les trois quarts l'écriraient ou le parleraient) et "l'avenir du français serait brillant" (Loquin, 1983).

Mais, pour d'autres, les plus nombreux semble-t-il à en juger d'après les divers congrès organisés sur l'avenir du français dans l'I.S.T., la situation est alarmante, et il faut réagir pour que le français soit lui aussi une langue scientifique internationale. Et, pour enrayer le déclin du français, une panoplie de mesures ont été proposées (notamment en 1981 au colloque de Montréal sur "L'avenir du français dans les publications et les communications scientifiques 
et techniques") et même déjà prises. Nous ne citerons que celles qui nous paraissent les plus importantes.

\section{MESURES COERCITIVES}

A l'échelle nationale, des mesures coercitives ont été préconisées. Les pouvoirs publics devraient, estime-t-on, n'accorder une aide financière à l'organisation de colloques internationaux que si ceux-ci font une place au français - en ayant recours éventuellement à la traduction - et aux chercheurs que si ces derniers présentent leur communication en français. La Ligue internationale des scientifiques pour l'usage de la langue française (créée au Québec en 1979) va jusqu'à suggérer d'obliger les chercheurs à publier en français en faisant de sorte que leur engagement, l'attribution de subventions, etc., soient fonction de leurs résultats scientifiques publiés en français (CLF, 1983, III, 255). De telles mesures coercitives n'ont jusqu'à maintenant guère eu d'effets. Une première circulaire du gouvernement français (1981) incitant les chercheurs français à publier leurs travaux en français et annonçant l'intention du gouvernement de refuser tout soutien technique et financier à des congrès qui se dérouleraient exclusivement en langue étrangère a suscité irritation et dédain (Lévy-Lebond, 1990), les chercheurs français refusant d'avoir à choisir entre subvention gouvernementale et publication internationale. En janvier 1990, le gouvernement français, au Forum de la communication technique et scientifique, a dû réitérer sa volonté de ne plus parrainer ni aider financièrement les rénions dont le français serait proscrit. 


\section{MESURES INCITATIVES}

À l'échelle nationale et internationale, il est souhaité que pouvoirs publics et milieux scientifiques et universitaires prennent conscience de la gravité de la situation (CLF, 1986) et incitent les chercheurs à publier dans leur langue. Il faudrait en particulier encourager la recherche de haute qualité puisque, comme l'affirmait Alfred Kastler de l'Académie des sciences de Paris - et Prix Nobel de physique en 1966 -, un article de haut calibre sera lu même s'il est rédigé en français (CFL, 1983, I, 281). Il faudrait aussi que les pays francophones produisent des revues de haute qualité. Dans les faits, depuis 1981, des progrès en ce sens ont été accomplis, essentiellement dans le cadre de la francophonie. En effet, le rôle de l'ensemble de la communauté francophone et des sommets réunissant les chefs d'État et de gouvernement des pays ayant en commun l'usage du français a été déterminant. On sait que trois sommets ont déjà eu lieu à Paris (1986), à Québec (1987) et à Dakar (1989), et des mesures concrètes ont été prises :

- Création de l'Université des réseaux d'expression française (UREF), dont la mise en oeuvre a été confiée à l'Association des universités partiellement ou entièrement de langue française (AUPELF). L'UREF entend contribuer au rayonnement international de la science en français notamment par la mise en relation d'équipes de chercheurs du Nord et du Sud, la constitution d'un espace francophone solidaire et par la création et la diffusion de I'I.S.T. en français. C'est ainsi que le gouvernement français a présenté à Dakar, l'an dernier, un projet de Cahiers de la recherche francophone qui fera partie du programme de 
I'UREF. Cette revue permettra aux chercheurs francophones du Nord comme du sud de diffuser le résultat de leurs recherches.

- Mise en place de réseaux de diffusion de revues scientifiques et de banques de terminologie et de néologie;

- Création d'un Fonds d'aide à la traduction et à l'interprétation dans les congrès scientifiques;

- Elaboration de réseaux informatisés de documentation scientifique et technique;

- Création de la Banque internationale d'information bibliographique sur les États francophones (BIEF).

La cooperation internationale a donc déjà porté fruit et il faut noter, à cet égard, le rôle déterminant joué par le Québec. En particulier, le colloque international organisé par le Conseil de la langue française en 1981, à Montréal, sur "L'avenir du français dans les publications et les communications scientifiques et techniques", et qui a réuni plus de 400 participants, a grandement favorisé la prise de conscience de la communauté scientifique francophone. Autre mesure concrète issue de la coopération : en 1985 a été lancée Médecine/Science, la première revue internationale de haut calibre en français et portant sur les sciences biomédicales. Cette revue, créée dans le cadre de la Commission permanente de coopération franco-québécoise, est indexée dans les Current Contents et tire aujourd'hui à près de 4600 exemplaires; cette entreprise, dont beaucoup doutaient au départ, démontre qu'il est faisable, tant au point de vue économique que scientifique, de diffuser la science en français. 
Il est essentiel que la concertation entre pays francophones se manifeste sur le plan de la terminologie. En effet, il faut non seulement créer des mots nouveaux au fur et à mesure que s'accroissent les connaissances - il est vain d'imposer l'usage du français s'il manque un grand nombre de mots dans notre vocabulaire technique et scientifique (Saint Robert, 1986, 89) -, mais aussi unifier la néologie pour que les mêmes mots nouveaux soient employés dans la quarantaine de pays francophones (Hagège, 1987, 284). C'est ce à quoi travaille le Conseil international de la langue française, chargé de maintenir l'unité de la langue et de contribuer à la mise à jour de la terminologie technique et scientifique, tâche que facilitera la mise sur pied de réseaux terminologiques francophones.

\section{SIMPIIFICATION DU FRANÇAIS}

Parmi les mesures envisagées, certaines concernent la langue employée par les chercheurs. Outre les appels en faveur d'une "modernisation de l'écriture du français" (Leconte, Cibois, 1989), on note des voeux plus réalisables dans l'immédiat. C'est ainsi que plusieurs souhaitent que les articles scientifiques soient jugés selon leur rigueur et leur clarté et non selon l'élégance de l'expression. Hagège $(1987,282)$ cite un professeur de l'Académie des sciences de Finlande qui écrit : "Un français simplifié [permettrait de] ralentir le déclin actuellement perceptible dans l'utilisation du français comme langue scientifique". Un professeur belge, Georges Thinès (CLF, 1983, III, 311-312), estime qu'il est "urgent de songer à une diffusion de la langue française largement indépendante des exigences de pureté". Et d'ailleurs, fait remarquer 
ce dernier, tous les scientifiques ne rédigent pas de façon parfaite. À son avis, il faut faire preuve de tolérance et favoriser l'utilisation d'un français pragmatique, sinon le français risque d'être éliminé de la compétition internationale. Il faudrait aussi, par voie de conséquence, faire une place à l'université à l'enseignement de la rédaction scientifique afin d'apprendre aux chercheurs à exposer avec rigueur et clarté le résultat de leurs recherches et à s'exprimer dans une langue "simple et dépouillée" (Hagège, 282).

\section{LUEURS D'ESPOIR}

Les mesures prises laissent croire à une prise de conscience réelle de la communauté scientifique francophone qui pourrait freiner le déclin du français dans les sciences et eviterait "un Yalta linguistique qui concéderait au français une certaine primauté historique dans les domaines des arts et des lettres, mais réserverait à l'anglo-américain, de manière quasi exclusive, la fonction d'exprimer la modernité technologique" ${ }^{4}$. Comme on I'a vu, tous les francophones ne se résignent pas à la disparition du français dans les sciences. Un événement récent constitue d'ailleurs un heureux présage : l'Institut Pasteur a publié, en mars 1990, le premier numéro d'un nouveau périodique de vulgarisation uniquement en français, Les Annales de I'Institut Pasteur. Actualités.

Cette parution et le succès remporté par la revue franco-québécoise Médecine/Science, les dispositions qui ont suivi le Sommet de Dakar de 1989 ainsi que la tenue du Forum de la communication scientifique et technique en janvier 1990 à Paris témoignent de la vitalité de la recherche scientifique de langue française et sont la preuve, comme l'affirmait déjà l'UREF en 
1987, qu'une communauté scientifique internationale utilisant le français est en émergence (UREF, 1987). on peut donc espérer que, grâce en particulier à la coopération internationale, de plus en plus de chercheurs francophones suivront les traces de Hubert Reeves, astrophysicien né à Montréal, ancien conseiller scientifique à la NASA, actuellement directeur du CNRS de France, dont les ouvrages rédigés en français connaissent un succès grandissant.

\section{NOTES}

1. Les chiffres donnés ci-dessous ne sont présentés qu'à titre indicatif. En effet, ils reposent sur différentes sources de données : Institute for Scientific Information (ISI), Centre national de la recherche scientifique (CNRS), etc.; de plus, les années de référence ne sont pas les mêmes puisque des statistiques n'existent pas pour les mêmes périodes.

2. CHI : Computer Horizon Incorporated, firme qui utilise l'index (SCI) de l'ISI.

NSF : National Science Foundation.

3. Dans tout le texte, nous avons désigné le Conseil de la langue française par le sigle CLF.

4. Journal officiel, 1.06.1989. 


\section{REFERENCES}

ASSOCIATION INTERNATIONALE DES PARLEMENTAIRES DE LANGUE FRANÇAISE (1980). Pré-rapport sur le français, langue scientifique présenté par la section française à la XI ${ }^{e}$ Assemblée générale, Ottawa, 8-12 septembre 1980 .

AQUIN, Thérèse (1987). "Le français, langue scientifique", Langue et société, $\mathrm{n}^{\circ} 21, \mathrm{p} .13$. BROGLIE, Gabriel de (1986). Le français pour qu'il vive, Paris, Gallimard.

BRUGUIÈre, Michel (1978). Pitié pour Babel, Paris, Nathan.

CASSEN, Bernard (1986). "Un nouveau front pour le français et les langues de l'Europe", Encrages, $n^{\circ}$ 16, pp. 12-14.

CONSEIL DE LA LANGUE FRANÇAISE (1983). L'avenir du français dans les publications et les communications scientifiques et techniques, Actes du colloque international tenu à Montréal du $1^{\text {er }}$ au 3 novembre 1981, Québec, Éditeur officiel, 3 vol.

CONSEIL DE IA LANGUE FRANÇAISE (1986). La place du français dans l'information technique et scientifique, Québec, Conseil de la langue française.

DECAUX, Alain (1990). "Enrayer la dynamique de la résignation", Le Monde diplomatique, janvier 1990, pp. 25 et 27 .

EDELMAN, Alex (1988). "Inside View of Pastor Institute", New Scientist, 22 oct. 1988 (reproduit dans Circuit, $n^{\circ} 25$, juin 1989, p. 10). 
HAGËGE, Claude (1987). Le français et les siècles, Paris, Odile Jacob.

HAUT CONSEIL DE LA FRANCOPHONIE (1987). État de la francophonie dans le monde. Données nouvelles 86-87. Paris, La Documentation française.

KOECHLIN, J.-B. (1988). "Le français, l'anglais, l'ordinateur... et les gens", dans Maurice Pergnier, éd., Le français en contact avec l'anglais, Paris, Didier Érudition.

LACANT, Jacques (1989). "Réflexions sur l'affaire des

"Annales de l'Institut Pasteur"", Défense de la langue française, $\mathrm{n}^{\circ} 148$, pp. 2-4.

"La France tente de contrer l'anglais dans les sciences", Le Devoir, 12 janvier 1990, p. 17.

LAPIERRE, Solange et Pierre Marchand (1989). "L'effet Pasteur ou le réflexe Coca-Cola", Circuit, $\mathrm{n}^{\circ} 25$, pp. $8-10$. 